\title{
Temperature and Density Mapping of Supersonic Jet Expansions Using Linear Raman Spectroscopy
}

\author{
G. Tejeda, B. Maté, J. M. Fernández-Sánchez, and S. Montero \\ Instituto de Estructura de la Materia, Consejo Superior de Investigaciones Científicas, Serrano 123, 28006 Madrid, Spain
}

(Received 21 July 1995)

\begin{abstract}
We present a first practical demonstration of Raman spectroscopy to obtain high definition maps of rotational temperatures and absolute densities in molecular supersonic jets.

PACS numbers: 33.20.Fb, 47.45.-n, 47.80.+v
\end{abstract}

Supersonic expansions associated to molecular gas jets are of considerable interest for various branches of physics, chemistry, and engineering. The properties of such rarefield environments differ markedly from those of a gas at thermodynamical equilibrium, and a complete diagnostic of their local properties is desired. The spatial distribution of molecular species, and its absolute density, the aggregation states (monomers, dimers, clusters, droplets, liquid, solid, aerosol, etc.), the translational speeds, the distribution of translational, rotational, and vibrational temperatures, and, in some cases, the anisotropic orientation of molecules along privileged directions are the main variables to be characterized.

Spectroscopic methods can provide answers to these questions, but none of them can be regarded as a complete, versatile, diagnostic tool for the supersonic jet. Here we stress the use of linear Raman spectroscopy, specially improved and adapted for this purpose, as a highly competitive diagnosis method capable of providing a wealth of data about the jet. As shown below, the mapping of several basic physical quantities of a supersonic expansion by means of Raman spectroscopy is nowadays an affordable methodology, with specific advantages over other spectroscopic methods, namely, (a) the universality (all molecules can in principle be studied), (b) a high spatial resolution (few microns), (c) linearity (Raman intensities are strictly proportional to the molecular density number), (d) a wide spectral range (1 to $6000 \mathrm{~cm}^{-1}$ ), and (e) a large dynamic range of intensities (over 6 orders of magnitude).

The feasibility of Raman spectroscopy in a supersonic expansion was demonstrated using a laser intracavity setup to compensate for the low efficiency of Raman scattering [1]. However, the critical optical alignment of the laser cavity has limited in practice a systematic and routine use of this possibility and, on the whole, Raman spectroscopy has received little attention as a diagnosis method for supersonic jets. Instead we use here a more flexible Raman setup whose efficiency is based on a global increase of sensitivity, derived from several instrumental improvements described next.

A 25-100 Jarrell-Ash monochromator has been converted to a multichannel spectrometer equipped with a 2380 lines/mm holographic grating as dispersive element.
It operates as a narrow band $\left(\leq 25 \mathrm{~cm}^{-1}\right)$ scanning single monochromator with a $512 \times 512$ pixel charge coupled device (CCD) detector refrigerated by liquid $\mathrm{N}_{2}$. The spectrometer covers a range of $7000 \mathrm{~cm}^{-1}$ at a maximum spectral resolution of $0.3 \mathrm{~cm}^{-1}$. The stray light rejection is good enough to record the good quality rotational spectra of gases needed to measure rotational temperatures. The detection limit in the gas phase is of the order of $10^{-3}$ mbar at a spectral resolution of $1 \mathrm{~cm}^{-1}$, i.e., between 2 and 3 orders of magnitude more sensitive than a standard Raman instrument.

An aluminum vacuum chamber of $42 \times 42 \times 30 \mathrm{~cm}^{3}$ contains the jet nozzle, a drill hole of $300 \mu \mathrm{m}$ diameter and $1 \mathrm{~mm}$ length, which can be accurately positioned along the $x, y, z$ directions. An optical multipass system for the laser exciting beam and the collection optics for the Raman signal are also placed inside the chamber. Ten independent remote controlled microactuators allow for the fine positioning of the optical components, and of the nozzle, with respect to the Raman focal point $P$ in Fig. 1. A field of the expansion of $25 \times 6 \times 6 \mathrm{~mm}^{3}$ can thus be monitored with an accuracy and a spatial resolution of a few microns. The laser beam is sharply focused onto the point $P$ of the jet, with a beam waist of about $14 \mu \mathrm{m}$, and is multipassed through the point $P$ by means of an

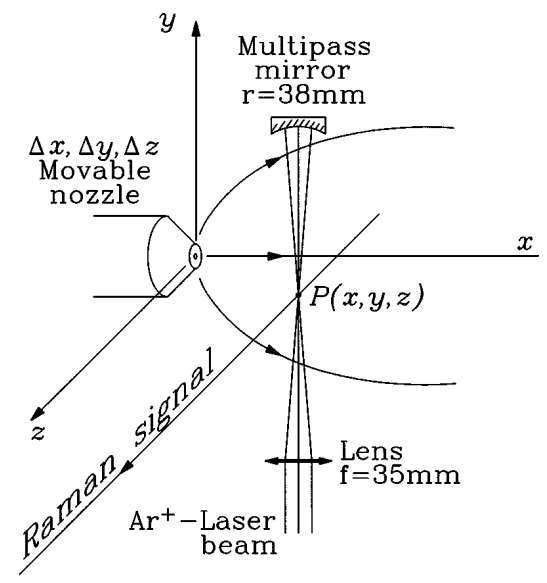

FIG. 1. Experimental setup for the observation of the Raman spectrum at points $P(x, y, z)$ of the supersonic jet, with $2 \mathrm{~W}$ laser power at $514.5 \mathrm{~nm}$. 
auxillary concave mirror, using the laser output mirror as a secondary reflector. Optical coupling between the multipass system and laser resonator is minimized by the mode mismatch due to the intermediate optical surfaces. The signal gain factor is about 5. Alignment is easy; it is not perturbed by the positioning motion of the nozzle, and the stability is maintained for periods of over $10 \mathrm{~h}$. The chamber is evacuated by means of two mechanical pumps in cascade. For a stationary continuous jet produced by the $300 \mu \mathrm{m}$ nozzle under a stagnation pressure of 1 bar, the residual pressure at the chamber is kept below 0.2 mbar. The stagnation pressure to generate the jet can be regulated between 0.5 and 8 bars.

The geometric structure and the local properties of a supersonic jet of polyatomic molecules cannot be described accurately by means of simple models. In the zone of silence of the jet an approximate description of the local properties is possible on the basis of the isentropic flow model of a perfect gas [2]. In this approach the local variables, temperature $(T)$, density number $(\mathcal{N})$, and modulus of the velocity vector $(|\mathbf{v}|)$ at point of the expansion axis downstream the nozzle are given in terms of the molecular mass $W$, of the coefficient $\gamma=C_{p} / C_{v}$ of heat capacities, and of the Mach number $M$, as $T=$ $T_{0} / F, \mathcal{N}=\mathcal{N}_{0} F^{1 /(1-\gamma)}$, and $|\mathbf{v}|=M\left(\gamma R T_{0} / W F\right)^{1 / 2}$, respectively; $T_{0}$ and $\mathcal{N}_{0}$ are the stagnation temperature and density number, respectively; $M=|\mathbf{v}| / a$ is the ratio of the local velocity $(|\mathbf{v}|)$ to the local velocity of sound (a) at the temperature $T$, and $F=1+M^{2}(\gamma-1) / 2 . M$ has been empirically modeled using the experimental data from large scale facilities (wind tunnels) in terms of the axial distance from the nozzle $(x)$ and of its diameter $(D)$. For an axisymmetric expansion [3]

$$
M=\tilde{x}^{\gamma-1}\left(b+c / \tilde{x}+d / \tilde{x}^{2}+e / \tilde{x}^{3}\right),
$$

for $\tilde{x}=x / D \geq 0.5$, with the coefficients

$$
\begin{gathered}
b=16.5404-15.8215 \gamma+4.7018 \gamma^{2}, \\
c=-15.6286+15.1459 \gamma-3.7335 \gamma^{2}, \\
d=13.1705-14.4312 \gamma+4.0591 \gamma^{2}, \\
e=-3.4116+3.7898 \gamma-1.0720 \gamma^{2} .
\end{gathered}
$$

Also off-axis density numbers are described by the empirical expression [4]

$$
\frac{\mathcal{N}(R, \theta)}{\mathcal{N}(R, 0)}=\cos ^{2}\left(\frac{\pi \theta}{17.0230-16.1334 \gamma+4.5346 \gamma^{2}}\right)
$$

where $R$ is the distance from the nozzle and $\theta$ the angle with the expansion axis. Combining the expressions, the off-axis properties for the local velocity and temperature can be obtained.

The expressions given above for $T, \mathcal{N}$, and $|\mathbf{v}|$ refer to an ideal unperturbed expansion propagating into a perfect vacuum. Real expansions differ from these ideal condi- tions by a residual pressure due to molecules not eliminated by the vacuum system. Collisions between the jet molecules and the residual molecules do substantially alter the structure of the expansion, as shown in the upper half of Fig. 2 (bold lines). A frontal shock wave (Mach disk) is produced at a distance $x_{M}=0.67 D\left(P_{0} P_{r}\right)^{1 / 2}$ [4,5] from the nozzle, where $P_{0}$ is the stagnation pressure and $P_{r}$ the residual pressure. The barrel shock wave confining the unperturbed regions of the jet has an approximate diameter of $0.75 x_{M}$, while the diameter of the Mach disk is about $0.5 x_{M}$. For real expansions Eqs. (1)(6) just hold for the zone of silence, of essentially laminar supersonic regime with $M \gg 1$, where the effect of the lateral and frontal shock waves is negligible.

A supersonic jet is usually a highly rarefield medium. For a stagnation pressure of 1 bar at $300 \mathrm{~K}$, the density number is $\mathcal{N}_{0}=2.5 \times 10^{25}$ molecules $/ \mathrm{m}^{3}$. For the test gas used here, $\mathrm{CO}_{2}\left(\gamma=1.3\right.$ at $\left.T_{0}=300 \mathrm{~K}\right)$, the density number falls down to $\mathcal{N}=2.3 \times 10^{21}$ molecules $/ \mathrm{m}^{3}$ at a distance $x=25 D$ downstream from the nozzle. Since the limit sensitivity of our system is about $\mathcal{N}=$ $2.5 \times 10^{19}$ molecules $/ \mathrm{m}^{3}$, the signal $/$ noise ratio of the recorded spectrum is good enough for quantitative work in a relatively wide region of the expansion.

The present setup has an excellent spatial resolution. The Raman spectrum is recorded from a cylinder-shaped region of diameter about $14 \mu \mathrm{m}$ in the $x-z$ plane, and height about $1.3 \mathrm{~mm}$ along the $y$ direction, centered at the point $P$ in Fig. 1. A longitudinal slice from this region can be selected by means of the entrance slit width of the spectrometer. The height of the observed slice along the $y$ direction can be controlled through the readout routine of the CCD array detector. In practice a spatial resolution of $7 \mu \mathrm{m} \times 300 \mu \mathrm{m} \times 14 \mu \mathrm{m}$, referred to the $x, y, z$ axes of Fig. 1, can be achieved under routine conditions $\left(T_{0} \geq\right.$ $300 \mathrm{~K}, P_{0} \geq 0.5$ bar, $D=300 \mu \mathrm{m}$ ) for most molecular gases. In addition, the positioning accuracy of the point $P$ is of the order of $\pm 1 \mu \mathrm{m}$, a spatial definition hard to attain with any other spectroscopic technique.

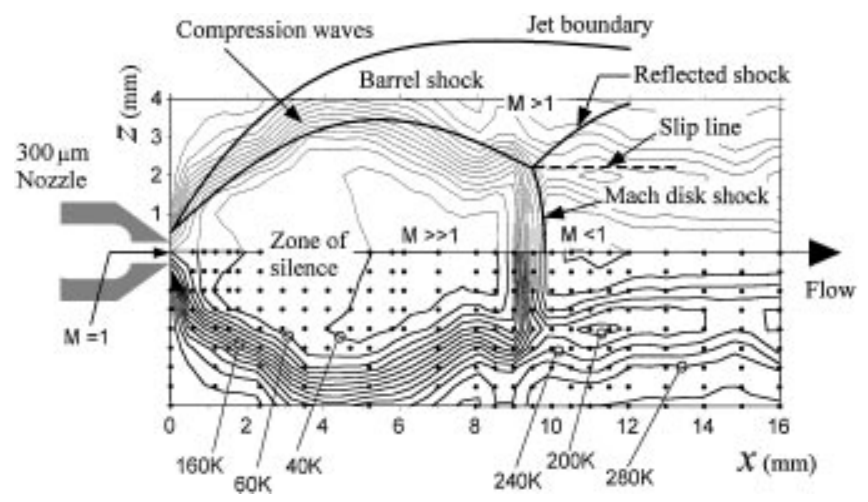

FIG. 2. Raman mapping of rotational temperatures in a supersonic jet of $\mathrm{CO}_{2}$ under a stagnation pressure of 2 bars. Isothermal lines are depicted at steps of $20 \mathrm{~K}$. 
Rotational temperatures $T_{R}$ at the points $P(x, y, z)$ of the jet are measured from the local Raman intensities $I_{J}(x, y, z)$ of the $J \rightarrow J+2$ transitions in the rotational spectrum, given for $\mathrm{CO}_{2}$ by

$$
\begin{aligned}
I_{J}(x, y, z)= & \mathcal{N} C \frac{(J+1)(J+2)}{(2 J+3)} \\
& \times \frac{\exp \left[-\beta B J(J+1) / T_{R}\right]}{\sum_{J}(2 J+1) \exp \left[-\beta B J(J+1) / T_{R}\right]},
\end{aligned}
$$

with $C$ a constant, $J$ the rotational quantum number $(J=$ even), $\beta=1.4388 \mathrm{~K} / \mathrm{cm}^{-1}$, and $B=0.39027 \mathrm{~cm}^{-1}$ the rotational constant of $\mathrm{CO}_{2}$. Equation (7) implies a Boltzmann distribution of population for the rotational levels. No deviation from this distributions has been observed in the nearly 200 rotational spectra recorded here between 40 and $300 \mathrm{~K}$. Vibrational temperatures $T_{V}$ at the points $P(x, 0,0)$ have been measured from the intensity ratio of the vibrational hot band of $\mathrm{CO}_{2}$ at $1409.5 \mathrm{~cm}^{-1}$ with respect to its fundamental counterpart at $1388.2 \mathrm{~cm}^{-1}$. Relative density numbers $\mathcal{N}$ and $\mathcal{N}^{\prime}$ between two points $P$ and $P^{\prime}$ are measured from the relative intensities of the vibrational band at $1388.2 \mathrm{~cm}^{-1}$ in the same experimental conditions. For vibrational temperatures $T_{V} \leq 300 \mathrm{~K}$ the ratio $\mathcal{N} / \mathcal{N}^{\prime}=I_{1388} / I_{1388}^{\prime}$ is a good approximation. Relative density numbers are converted to absolute density numbers by comparing the intensity at a reference point of the jet with the intensity recorded under static conditions at a pressure of 200 mbar.

To test the mapping capabilities of our setup, a sharp shock wave structure with a Mach disk at $10 \mathrm{~mm}$ from the nozzle was created by rising the residual pressure in the expansion chamber. The temperature and density distributions of this structure have been investigated here.

A map of rotational temperatures of the $\mathrm{CO}_{2}$ molecules expanding in the jet is shown in Fig. 2. The pure rotational Raman spectra in the range 12 to $32 \mathrm{~cm}^{-1}$, covering the transitions $J=8 \rightarrow 10$ to $J=16 \rightarrow 18$ were recorded at points $P(x, 0, z)$ of the $x-z$ symmetry plane of the expansion at steps of 0.5 or $1 \mathrm{~mm}$, for $0<$ $x \leq 22 \mathrm{~mm}$ and $0 \leq z \leq 4 \mathrm{~mm}$. Solid dots at the lower part of Fig. 2 correspond to the sites where such spectra were recorded. The rotational thermal data were obtained from Eq. (7) and were then fitted to constant temperature surfaces. The $x-z$ section of these surfaces is shown in the map in Fig. 2 for $x \leq 16 \mathrm{~mm}$, the region where the more prominent features have been observed. The characteristic features of the Mach disk and of the lateral shock waves can easily be recognized. The bold lines in the upper part of Fig. 2 display a typical supersonic jet as described in the literature [3,5], scaled for the position of the Mach disk at $x_{M}=10 \mathrm{~mm}$, the value expected for the present stagnation and residual pressures of 2 bars and 0.81 mbar, respectively. As can be seen from Fig. 2, the rotational rethermalization process is quite sharp at the shock waves, with rotational temperature gradients in the neighborhood of the Mach disk of about $200 \mathrm{~K} / \mathrm{mm}$ and of $100 \mathrm{~K} / \mathrm{mm}$ at the lateral barrel shock region. On the contrary, the evolution of vibrational temperatures with the expansion is far smoother and remains "frozen" at $T_{V} \approx 180 \mathrm{~K}$ for $x \geq 6 \mathrm{~mm}$, with no evidence of rethermalization across the Mach disk.

For the same expansion, an absolute density number map in the domain $(5-100) \times 10^{21}$ molecules $/ \mathrm{m}^{3}$ is shown in Fig. 3. It was generated from the Raman intensity of the vibrational line of $\mathrm{CO}_{2}$ at $1388.2 \mathrm{~cm}^{-1}$, measured at the sites marked by a solid dot in the lower part of the figure. The $x-z$ section of the constant density surfaces fitted to the experimental data is shown in Fig. 3. Again the Mach disk expected at $x_{M}=10 \mathrm{~mm}$ and the lateral barrel compression shock waves can be clearly identified in the map. An increase of density by a factor of about 6 is observed at the shock waves, which is comparable to the relative rotational thermal jump between homologous points. In general, the spatial distribution of rotational temperatures and of molecular density number are correlated, but some distinctive features deserve mention: Near the slip line in Fig. 2 there is a cold channel with rotational temperatures about $40 \mathrm{~K}$ lower than the already rethermalized surrounding. Behind the Mach disk in Fig. 3 there is a spot of strong compression, located roughly between the slip line and the reflected shock wave, where the relative density number increases by a factor of 8, apparently with no important variation of the temperature.

The density number data obtained here are not too different from those obtained in large scale facilities, mainly wind tunnels. The unperturbed region of the expansion where supersonic conditions prevail (zone of silence) is shown in detail in Fig. 4. The constant density number lines predicted according to Eqs. (1)-(6) are shown in thin lines, while the present experimental results are in bold lines in the lower part of the picture. For distances up to $7 \mathrm{~mm}$ from the nozzle the agreement

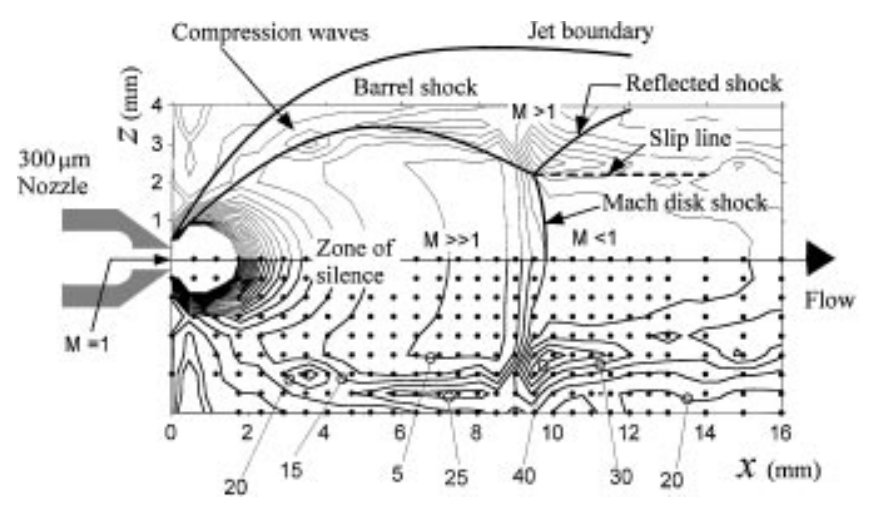

FIG. 3. Raman mapping of the absolute density number of molecules in a supersonic expansion of $\mathrm{CO}_{2}$. Constant density number lines are depicted at steps of 5 units, in units of $10^{21}$ molecules $/ \mathrm{m}^{3}$. Same conditions as in Fig. 2 . 


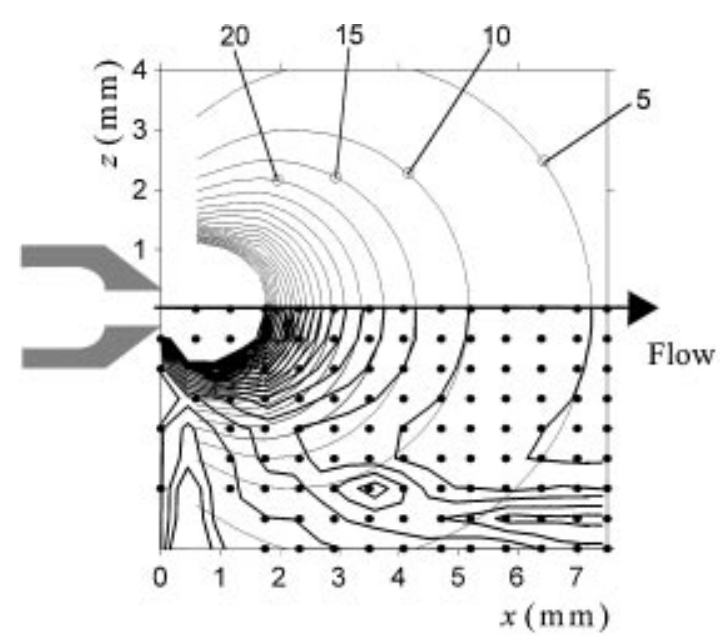

FIG. 4. Experimental absolute density number of molecules (bold lines) compared to the distribution predicted from Eqs. (2)-(6) (thin lines). Units of $10^{21}$ molecules $/ \mathrm{m}^{3}$. Same conditions as in Fig. 2.

is fairly good on the expansion axis, but the angular behavior predicted by Eq. (6) differs from the observed data for angles $|\theta|>30^{\circ}$ off-axis, where the effect of the frontal and lateral shock waves starts to be noticeable. In spite of the nonideal shape of the nozzle used here, and of the different scale of experiments [4] to obtain the coefficients in Eqs. (2)-(6), the overall agreement is better than expected.

As far as the rotational temperatures are concerned, we notice an increase of about $20 \mathrm{~K}$ with respect to the theoretical predictions of the isentropic approximation, in agreement with previous Raman experiments in the expansion axis [6]. This has been attributed to condensation effects. Indeed the liquid phase band of $\mathrm{CO}_{2}$ at $1385.8 \mathrm{~cm}^{-1}$ is observed in our spectra in the zone of si- lence, with a relative intensity of up to $3 \%$ with respect to that of the gas phase $Q$ branch at $1388.2 \mathrm{~cm}^{-1}$.

The scope of Raman spectroscopy for hypersonic research is shown with the present results. It is worth mentioning that the total recording time of the nearly 200 spectra required to generate the data points for the maps of Figs. 2 and 3 has amounted to $6 \mathrm{~h}$ per map. This performance opens the possibility for systematic quantitative studies of many basic aspects of the physics of supersonic expansions at a molecular level, beyond the hypothesis of the continuum. Collisions, flow field, and nonequilibrium are some of the questions where contributions can be expected. Together with the purely spectroscopical applications for the simplification of molecular rovibrational spectra of gases at low temperature, for the observation of frozen conformers of polyatomic molecules, or for the investigation of aggregation states, the present methodology opens appealing possibilities in basic as well as in applied research.

The Spanish DGICYT is acknowledged for financial support (Research Project No. PB91-0133).

[1] I. F. Silvera and F. Tommasini, Phys. Rev. Lett. 37, 136 (1976).

[2] M. J. Zucrow and J. D. Hoffman, Gas Dynamics (Wiley, New York, 1976), Vol. I.

[3] D. R. Miller, in Atomic and Molecular Beam Methods, edited by G. Scoles (Oxford University Press, New York, 1988), Vol. I.

[4] H. Ashkenas and F. S. Sherman, 4th International Symposium on Rarefield Gas Dynamics 2, 84 (1964), Sec. 7.

[5] K. Bier and B. Schmidt, Z. Angew. Phys. 13, 493 (1961).

[6] G. Luijks, S. Stolte, and J. Reuss, Chem. Phys. 62, 217 (1981). 